\title{
Performance Review of PAPR Reduction Techniques in OFDM Systems
}

\author{
Shiwani Sandal* and Lavish Kansal \\ School of Electronics and Electrical Engineering, Lovely Professional University, \\ Phagwara - 144411, Punjab, India; \\ shiwanisandal@gmail.com, lavish.15911@lpu.co.in
}

\begin{abstract}
Orthogonal Frequency Division Multiplexing (OFDM) has great demand in wideband digital communication. It encodes the data on multiple carrier frequencies. Its application areas are digital audio broadcasting and high definition television, wireless network, 4G mobile communications. Due to its great advantages like it provides high spectral efficiency, remove inter symbol interference, reduced nonlinear distortion it is widely used for communication purpose. Major problem of this scheme is high Peak to Average Power Ratio (PAPR). To overcome this problem i.e. PAPR, here in this paper we figure out different reduction techniques, there pros and cons and also find Selective Mapping (SLM) is better technique due to its several advantages with reduced PAPR and better Bit Error Rate (BER) which is discussed further in this paper.
\end{abstract}

Keywords: Hybrid Techniques, OFDM, PAPR, Precoding Techniques, Reduction Techniques

\section{Introduction}

Lately, the necessity of multimedia wireless data services has grown extravagantly which bring the communication in the era of 4 th generation wireless communication system. In this era where number of users are more with restricted bandwidth, the need of modern digital wireless communication is adopted because it provides better spectral efficiency with efficient bandwidth and also robust to multipath channel environment which is known as multi-carrier communication system. This type of communication system provides an ease of high speed data rate at cheap cost for many users with high reliability. The main difference between single carrier system and multi carrier system is that in single carrier system, single carrier occupies entire communication bandwidth but in multicarrier available bandwidth is distributed among many sub-carriers so that each subcarrier carries the use- ful amount of bandwidth according to its need as compare to the whole bandwidth as in case of single carrier system. These enormous features of multicarrier system draw our attention to study Orthogonal Frequency Division Multiplexing (OFDM). OFDM is the base for all $4 \mathrm{G}$ wireless communication systems because it has large capacity (number of subcarriers), high data rate (excess of 100 Mbps) and efficient use of bandwidth, receiver simplicity and ubiquitous coverage with high mobility. As we know OFDM system is a multi-carrier modulation system in which through serial-to-parallel port, input data stream converts into $\mathrm{N}$ parallel data stream. Due to the closeness of subcarriers they can sees flat faded channel. The process of modulation and demodulation in OFDM is done by fast DSP applications (FFT/IFFT). After the generation of parallel symbol, modulation is done on each stream and they carried at unique central frequencies and they must be orthogonal to each other. This task which is most

${ }^{*}$ Author for correspondence 
powerful features of OFDM i.e. orthogonality is done by using IFFT. To remove interference between the successive OFDM symbols guard bands are inserted. Three methods are there for this purpose which are cycle suffix, cycle prefix and zero padding. Also one more advantage of adding guard band is that OFDM convert wideband frequency selective channel into collection of parallel narrow band flat fading channel in which one channel across each subcarrier is there.

Applications of OFDM system are:

- OFDM has wide range of application in Digital Audio Broadcasting (DAB) used in several countries especially in Europe.

- It is used in Digital Broadcast Services to handheld services.

- It is used in LTE and LTE advanced as it gives higher performance, better spectral efficiency, reduced cost, efficient services and better quality of service.

- It is used in High-Definition Television (HDTV).

- It has wide range of application in wireless LAN.

- Mobile Broadband Wireless access technologies also include in the application of OFDM.

Despite of many advantages and application areas, the major problems in OFDM system is PAPR, BER and synchronization at the receiver which restrict the OFDM application areas to some extent. So to overcome the main problem i.e. PAPR we have many reduction techniques, precoding techniques, different transform and the hybrid technique which are discussed further.

To reduce the PAPR in OFDMA system a reduction technique named as the interleaved portioning PTS scheme is efficient which is inferior than the adjacent PTS scheme because in IP-PTS candidates generated in IP-PTS is not fully independent ${ }^{1}$. Another method for reducing the PAPR is a novel PAPR reduction algorithm based on tree structured searching technique in which PTS scheme is used although it becomes impractical solution at high data transmission rate but proposed solution is also given to overcome this problem $²$. A new technique named as Reduced Complexity SLM (RC-SLM) is proposed in which assigning power to the different subcarriers using an unequal power distribution strategy in which computational complexity is lower as compared to other and lower the PAPR of a system $\stackrel{3}{*}$. Another method is a companding scheme which is better than the conventional companding, SLM, PTS because it reduces the PAPR in better way and also without any altering the original signal ${ }^{4}$. A new simple and very efficient technique is proposed which is based on autoregressive filtering that can lower the PAPR as well as BER performance of OFDM signal. Also it is very efficient for any type of modulation scheme ${ }^{5}$. Using a single technique we can also use the combination of PTS and SLM technique to reduce PAPR with less side band information but it is not beneficial for the system having more sub channel and high stability is required ${ }^{6}$. Also combination of SLM and clipping technique is used as clipping reduced the clipping noise and SLM giving the better performance of OFDMA signal by reducing the PAPR at efficient level ${ }^{7}$. Another combination is a novel clipping and filtering based on clipping noise cancellation method is proposed which reduces the PAPR as well as in and out band distortion ${ }^{\circ}$. To lower the PAPR precoded technique named as ZCMT precoded multi-carrier OFDM system is proposed given in which ZCMT precoded matrix is used before IFFT to reduce PAPR . Another precoding method is hadamard precoding is used in OFDM system and PAPR is compared between simple OFDMA systems and using this technique and the result is $1.5 \mathrm{~dB}$ is reduced by using this technique ${ }^{10}$. Hybrid techniques which is the combination of block coding scheme and DCT is proposed to reduce the PAPR in OFDM system. With this algorithm PAPR gets lowered, error correction is there, noise immunity is there ${ }^{11}$. Also a new grouped precoding technique based on DCT transform is used to lower the $\mathrm{PAPR}^{12}$. Another hybrid technique is Tone Reservation (TR) technique with DCT transform to reduce the PAPR. This hybrid technique is good because it reduce complexity as compared to FFT as well as with this method BER performance cannot be effected ${ }^{\underline{13}}$. Also if we use the combination of DCT-PRECODING with clipping as compared to the DCT precoding technique then it better reduces the PAPR of OFDMA system ${ }^{14}$. Another beneficial technique is the post coding scheme which is based on DCT transform is employed after the IFFT to reduce 
the PAPR of OFDM signal and also improves the BER performance ${ }^{15}$.

\section{System Description}

OFDM is a MCM system which transmits signals on multiple subcarriers having unique frequencies and must be orthogonal to each other. OFDM has great importance in both wired as well as wireless communication, such as the Asymmetric Digital Subscriber Line (ADSL) and the IEEE 802.11 standard. The OFDM system is shown in Figure 1 consists of transmitter, channel and receiver blocks. If the data is transmitted at high rate, the bandwidth of the channel becomes wide and may exceed the coherence bandwidth of the channel. This distorts the signal and causes Inter Symbol Interference (ISI). A guard interval is introduced in every OFDM symbol to eliminate ISI. Two approaches are followed in OFDM to insert guard interval. The first one is known as zero padding, where zeros are inserted between OFDM symbols. The second one is known as cyclic prefix, where a part of the end of the OFDM symbol is copied and place in the start-

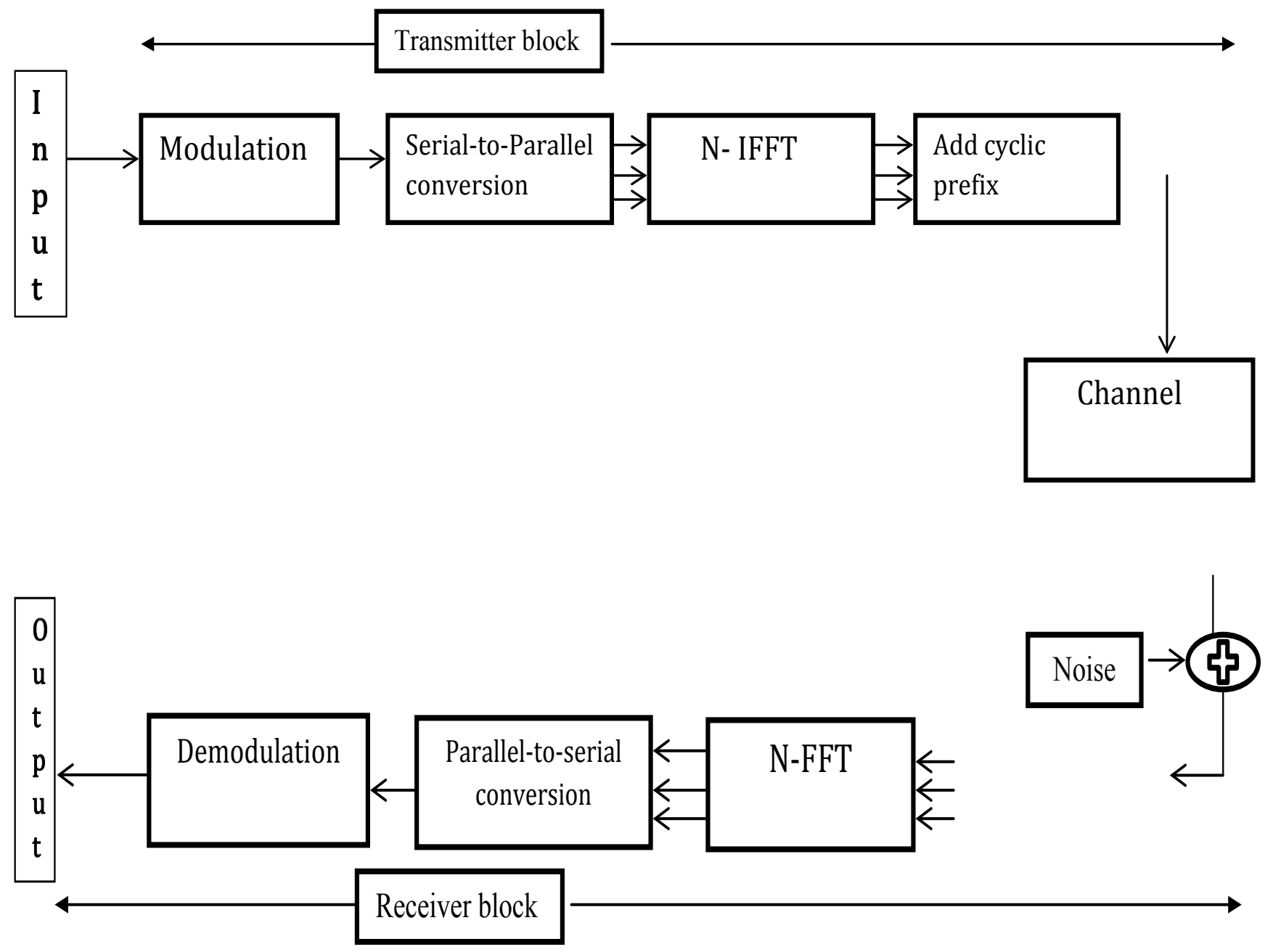

Figure 1. Block diagram of OFDM system. 
ing of the next OFDM symbol in time domain. The length of the guard interval must be at least equal to the length of the channel response to avoid the ISI. Usage of cyclic prefix is preferred than zero padding when FFT is used. Because FFT algorithms require the signal to be periodic to produce accurate results and cyclic prefix makes the signal periodic. Figure 1 shows the block diagram of OFDM system.

OFDM symbols are generated as follows. The transmitter section in which digital data which is to be transmitted firstly convert into a mapping of subcarriers amplitude and phase using different modulation techniques i.e. BPSK, QPSK or QAM. After the modulation, modulated data is converted into parallel data stream for efficient and faster utilization of bandwidth. For representing that modulated data into Time domain is created with the help of an Inverse Fast Fourier Transform (IFFT) which is useful method to implement DFT. To extend the time domain data is done by the addition of cyclic prefix to each symbol that become helpful to solve ISI as well as Inter carrier interference problems. Then this signal is passed through the channel. At the receiver section, the opposite process of transmitter is done i.e. the cyclic prefix is removed and converted the time domain signal into frequency domain with the help of FFT. At the last, the parallel data is converted back into serial data.

\section{PAPR and its Reduction}

One new problem that comes in OFDM systems is PAPR. The main reason of occurring of PAPR is that there is uniform power spectrum at the input symbol stream of IFFT but at the output side non uniform spectrum is there. PAPR is defined as the peak signal power versus the average signal power. The PAPR of a signal is expressed by the following formula:

$$
P A P R_{d b=} 10 \log \left(\frac{\max x(t) x *(t)}{E x(t) x *(t)}\right)
$$

Where ()$^{\star}$ represents the conjugate operator.

\subsection{Impact of High PAPR on a OFDM System}

OFDM main problem is high PAPR because when OFDM signal transmits over an optical fiber channel, due to high PAPR distortions is there caused by nonlinear devices, such as analog to digital converter, transmission fiber etc. Large PAPR results in band distortion as well as out of band radiation are the major problems suffered by transmitted OFDM signal. Also it increases the difficulty for analogto-digital converter and digital-to-analog converter. It also reduces the efficiency of radio power amplifier. The main reason of occuring the PAPR is that in multicarrier system different subcarriers are out of phase with each other. At every instant they exhibit different phase value. When points achieve maximum value simultaneously then this will result that the output envelope suddenly to shoot up and becomes the reason of causing peak in the output envelope. In multicarrier system many modulated subcarriers are there so more peak value will be there as compared to the average of the whole system. This ratio is called PAPR. Due to large PAPR power amplifier should be operated with large power back-offs results in expensive transmitters and inefficient amplification will cause.

\subsection{PAPR Reduction Techniques}

\subsubsection{Signal Scrambling Techniques}

The basic idea behind this technique is that it selects the sequence of signals with least PAPR value. The techniques come under this category are SLM, PTS and Interleaved OFDM.

\subsubsection{Selected Mapping}

Technique is a scrambling technique in which a set of signals are transmitted which representation of some information in each among the transmitted signals a very minimum PAPR value over the channel. The selected signals sent to the receiver along with Selected Signal as Information (SSI) in order to decode information at the receiver. Even though SLM method uses codes it's been 
limited for only PAPR reduction but not any error correction codes.

\subsubsection{Partial Transmits Sequence (PTS)}

The PTS is most popularly used method in reduction of PAPR. The idea behind the PTS scheme is that original sequences of OFDM divided into diverse sequences and multiplies each sequence by distinct weights. The weights which offer better improvement in OFDM signal with less PAPR is the best results.

\subsubsection{Interleaved OFDM}

The method is like alternate of SLM technique, just the difference is that it uses an interleaver instead of using the sequences of phases. Interleaver is a computational device which has specific manner permutation and operates on $\mathrm{N}$ symbol block. The interleaved OFDM's block diagram interleavers and de-interleavers are usually denoted by the symbol $\pi$ and $\pi-1$.

\subsubsection{Signal Distortion Techniques}

There is different type of reduction techniques that comes under signal distortion technique. The concept behind these techniques is that it distorts the original OFDM signal non linearity.

\subsubsection{Clipping and Filtering}

In this technique it clips the level to those signal components that exceed some unvarying amplitude called clip level. It is very effective as well as very simple technique. But the main problem suffered in this is that due to clipping distortion power is there which is known as clipping noise and it also expands the spectrum of transmitted signal which results in interference. It is nonlinear process so it cause in band noise distortion and also degrades the performance of BER. It also reduces the spectral efficiency because of out of band noise. Filtering can also result in same i.e. spectrum growth but it reduce the out of band radiation after clipping but may cause some peak regrowth, which the signal of peak exceeds in the clip level. The combination of clipping and filtering reduces the PAPR without any expansion in spectrum but computation complexity is there in OFDM transmitter. Also we have to perform interpolation before clipping to prevent out of band radiation. But it causes peak re-growth. So iterative clipping and frequent domain filtering is used to remove or prevent the peak re-growth.

\subsubsection{Tone Reservation (TR)}

In this technique we keep a small set of tones for reducing the PAPR. It is a method in which data block added and time domain signal. Data block is dependent time domain signal to the original multicarrier signal to reduce the high peak. At the transmitter of system the time domain signal can be calculated simply and stripped off at the receiver. It depends upon number of reserved tones, complexity that the exhibit, location of reserved tones and allowed power on reserved tones to how much we can reduce the PAPR.

\subsubsection{Tone Injection (TI)}

It is based on additive method for reducing the PAPR. Using this technique we can reduce PAPR without any loss of data rate. It uses a set of equivalent constellation points for an original constellation points to lower the PAPR. The main method is to increase the size of constellation.

\subsubsection{Peak Windowing}

Here, we can remove large peaks but at the slight cost of self-interference when large peaks arise infrequently. The main disadvantage is that BER increases and out of and radiation increase. The only difference between clipping and peak windowing is that we get better spectral efficiency in case of peak windowing. In peak windowing method we multiply peak of large signal with specific window for example Kaiser window, hamming window, Gaussian shaped window.

\subsubsection{Companding Transform}

This technique is a combination of compression and expansion which is basically applicable for audio signals. The idea behind this technique is that after companding lower peak values are increased but higher remains constant hence the peak of OFDM is increased resultantly the PAPR reduces. The drawback of this technique is that the average power needs to be maximized to decrease the PAPR. This puts more burdens on transmitter relative with the average power. 


\subsubsection{Coding Techniques}

The basic idea behind this technique is that it uses set of codes to reduce the PAPR. The code words are used before the application of IFFT. "When $\mathrm{N}$ signal are added with same phase they produce peak power which is $\mathrm{N}$ times greater than the average power". This doesn't do out band radiations and creates Do distortion signal but due the reason it uses set of code words. The bandwidth efficiency is lesser when code rate is reduced. Complexity with this type of technique is that it needs to find best and suitable code words for reduction of OFDM and need to maintain the look up tables for coding and decoding.

\subsubsection{Precoding Techniques}

Discrete-Cosine Transform Matrix (DCTM) precoding method is goof for improving PAPR as well as BER. In this technique constellation symbols are precoded with linear independent precoder, which diminish the autocorrelation relationship of the IFFT input sequence and disperse the information among subcarriers. Other transforms that are being used for the purpose of precoding are DCT, DST, DWT, etc.

\subsubsection{Hybrid Techniques}

It includes the combination of precoding and reduction techniques (signal scrambling and signal distortion).

\subsubsection{Reduction with Transform}

The combination of DCT precoding with clipping can lowered the PAPR in very efficient manner. Firstly, by DCT data is transformed into new modified form. Secondly, the proposed scheme utilizes the clipping method. In this scheme first the transmitted data are transformed with the help of DCT precoding matrix before IFFT operation, which reduces the PAPR. After that, clipping is done.

\section{Conclusion}

In this paper we discussed various PAPR reduction techniques and find SLM is the best techniques because RC-SLM (Reduced Complexity Selective Mapping) is a proposed scheme which reduced complexity and improves the performance of PAPR about $0.3 \mathrm{~dB}^{16}$. Also novel SLM in which linear feedback shift register circuit and $\mathrm{m}$-sequence named MSLM technique is used which results to lowered the PAPR as shown in results ${ }^{17}$. The integrated SLM technique consisting of Reed-Muller codes, Constellation scheme termed as RE-CE-SLM also improves PAPR as discussed in the simulation results ${ }^{18}$.

\section{References}

1. Wu X, Wang J, Mao Z, Zhang J. Conjugate interleaved partitioning PTS Scheme for PAPR reduction of OFDM signals. Circuits, Systems and Signal Processing. 2010 Jun; 29(3):499-514.

2. Lee BM, de Figueiredo RJP, Kim Y. A computationally efficient tree-PTS technique for PAPR reduction of OFDM signals. Wireless Personal Communications. 2012 Jan; 62(2):431-42.

3. Hassan ES, Xu Z, El-Khamy SE, Dessouky MI, El-Dolil SA, Abd El-Samie FE. Peak to Average Power Ratio reduction using Selective Mapping with unequal power distribution. Journal of Central South University. 2012 Jul; 19(7):1902-8.

4. Renze L, Longjiang J, Lang L, Jie L, Weile Z. Reducing the Peak to Average Power Ratio of OFDM system with low complexity. Journal of Electronics (China). 2006 Jan; 23(1):26-8.

5. Hasan MM. PAPR reduction in OFDM systems based on autoregressive filtering. Circuits, Systems and Signal Processing. 2014 May; 33(5):1637-54.

6. Duanmu C, Chen H. Reduction of the PAPR in OFDM systems by intelligently applying both PTS and SLM algorithms. Wireless Personal Communications. 2014 Jan; 74(2):849-63.

7. Lee BM, Kim Y, de Figueiredo RJP. Performance analysis of the clipping scheme with SLM technique for PAPR reduction of OFDM signals in fading channels. Wireless Personal Communications. 2012 Mar; 63(2):331-44.

8. Chakrapani A, Palanisamy V. A novel clipping and filtering algorithm based on noise cancellation for PAPR reduction in OFDM systems. Proceedings of the National Academy of Sciences, India Section A: Physical Sciences. 2014 Sep; 84(3):467-72.

9. Baig I, Jeoti V. A new DCT matrix precoding based RI-OFDMA uplink system for PAPR reduction. 4th International Conference on Intelligent and Advanced Systems; 2012 Jun. p. 680-4.

10. Wang ZP, Xiao JN, Li F, Chen L. Hadamard precoding for PAPR reduction in optical direct detection OFDM systems. Optoelectronics Letters. 2011 Sep; 7(5):363-6. 
11. Hsu CY, Do HG. The new Peak to Average Power reduction algorithm in the OFDM system. Wireless Personal Communications. 2007 Jun; 41(4):517-25.

12. Wang ZP, Zhang SZ. Grouped DCT precoding for PAPR reduction in optical direct detection OFDM systems. Optoelectronics Letters. 2013 May; 9(3):213-6,

13. Zhou K, Zhang J, Xiao L. A research on improving the performance of OFDMA system by using DCT/IFFT structure. The 2nd International Conference on Information Science and Engineering; 2010 Dec. p. 1766-9.

14. Wang ZP, Chen SF, Zhou Y, Chen M, Tang J, Chen L. Combining discrete cosine transform with clipping for PAPR reduction in intensity-modulated OFDM systems. Optoelectronics Letters. 2014 Sep; 10(5):356-9.
15. Chen FN, Wang ZP. A post-coding scheme for Peak to Average Power Ratio reduction in intensity modulated optical OFDM systems. Optoelectronics Letters. 2014 Jul; 10(4):295-8.

16. Hassan ES, Zhu XU, El-Khamy SE, Dessouky MI, El-Dolil SA, Abd El-Samie FE. Peak to Average Power Ratio reduction using selective mapping with unequal power distribution. Journal of Central South University. 2012 Jul; 19(7):1902-8.

17. Meymanatabadi S, Niya JM, Mozaffari B. Selected mapping technique for PAPR reduction without side information based on m-sequence. 2013 Aug; 71(4):2523-34.

18. Liang HY. Integrating $\mathrm{CE}$ and modified SLM to reduce the PAPR of OFDM systems. Wireless Personal Communications. 2015 Jan; 80(2):709-22. 